\title{
FATORES DE SUCESSO DE UMA INOVAÇÃO NO CONTEXTO UNIVERSITÁRIO: UM ESTUDO DE CASO DO SETOR PECUÁRIO
}

\author{
Success factors of an innovation process in the university context: \\ a case study of cattle sector (Starea)
}

\author{
Dany Flávio Tonelli', André Luiz Zambalde²
}

\begin{abstract}
RESUMO
O tema deste trabalho é o processo de inovação no contexto universitário. Objetivou-se, por meio da investigação de um caso específico de inovação no setor pecuário (Amiréia), identificar os fatores essenciais que proporcionaram a aplicação dos conhecimentos desenvolvidos em pesquisas científicas no mercado. A metodologia foi o estudo qualitativo de caso. As técnicas utilizadas foram entrevistas e análise documental. Os resultados permitiram a identificação de três fatores que foram essenciais para o sucesso da inovação. Esses fatores são: o conhecimento científico prévio acumulado; a importância de parcerias entre grupos de pesquisa e empresas e a cultura disseminada no mercado.
\end{abstract}

Termos para indexação: Pesquisa, desenvolvimento, aplicabilidade, processo de inovação.

\section{ABSTRACT}

The subject of this work is the innovation process in the university context. The purpose was, by means of an investigation of a specific innovation case in the cattle sector (Starea), to identify the essential factors that promote the transference of knowledge developed by scientific researches to the market. The methodology was a qualitative study of case. The techniques were: interviews and documental analysis. The results led to the identification of three factors that were essential to the success to the innovation. These factors are: a) the previously accumulated scientific knowledge; b) the importance of partnership between groups of research and enterprises and c) the culture disseminated in the market.

Index terms: Research, development, applicability and process of innovation.

(Recebido em 14 de março de 2007 e aprovado em 13 de junho de 2008)

\section{INTRODUÇÃO}

Com o advento da economia baseada no conhecimento, a questão dos resultados obtidos com a pesquisa científica foi colocada no centro do debate público e tornou-se preocupação chave dos governos. Tendo em vista que a produção de conhecimento científico, por si só, não representa garantia de aplicação, o foco recaiu sobre os processos de inovação. No passado, as políticas governamentais pautavam-se no conceito linear do processo. Atualmente, elas reconhecem a importância da interação e da complementação entre diversos atores institucionais e distintas necessidades.

Objetivou-se, no presente trabalho, investigar um caso de inovação no setor agropecuário, ocorrido no contexto universitário, apontando os fatores críticos considerados essenciais para o processo de articulação dos resultados da pesquisa até o desenvolvimento do produto e a sua comercialização.
Inicialmente, realiza-se uma abordagem sobre $\mathrm{o}$ atual contexto institucional que impõe mudanças no comportamento das universidades, em relação ao conhecimento científico nelas produzido. Posteriormente, aborda-se a dinâmica dos processos de inovação tidos como essenciais para a transformação de conhecimentos em produtos. Na seqüência, apresenta-se a metodologia utilizada, seguida pelos resultados e discussões e, consequientemente, pela conclusão.

De um lado, o desenvolvimento de conhecimento científico e, de outro, a busca pela aplicabilidade do mesmo. Nunca se falou tanto sobre esse assunto como na época atual, especialmente em razão da recente Lei Nacional de Inovação. $\mathrm{O}$ atual contexto enaltece a preocupação com a aplicabilidade comercial e a potencialidade de inserção social dos conhecimentos alcançados pelas pesquisas, dando a elas um sentido econômico e aumentando, assim, a busca pelo desenvolvimento da sociedade.

Brito (2000) afirma que a síntese dessas transformações seria o surgimento de novas formas

${ }^{1}$ Administrador, Doutorando em Administração - Rua José Junqueira Garcia, 42 - Santa Filomena - 37200-000 - Lavras, MG - danytonelli@gmail.com 2Engenheiro de Telecomunicações, Ph.D. em Ciência da Computação, Professor - Departamento de Ciência da Computação/DCC - Universidade Federal de Lavras/UFLA - Cx. P. 3037 - 37200-000 - Lavras, MG - zamba@ufla.br 
organizacionais que passariam pela redefinição da relação existente entre o público e o privado. Nesse novo ambiente, a preocupação com a propriedade intelectual e a geração de tecnologias comercializáveis transita ao lado da articulação de parcerias com a iniciativa privada e da diversificação das fontes de financiamento de projetos. Dessa forma, está implícita a existência de uma pressão por modificação do comportamento organizacional na direção da obtenção de maior legitimidade social.

Diversos estudos têm revelado a preocupação com essas transformações (ETZKOWITZ \& LEYDESDORFF, 1995, 1997, 2000; GIBBONS et al., 1994; KNORR-CETINA, 1982; LATOUR \& WOOLGAR, 1997). Em síntese, esses estudos preconizam a necessidade de buscar-se uma conexão mais sólida entre a produção de conhecimento e a sua aplicação prática.

No que se refere à ligação entre aplicabilidade do conhecimento científico e o desenvolvimento social e econômico dos países, ela tem sido considerada como fórmula segura para traçar uma trajetória promissora de crescimento. Schumpeter (1942) já afirmava que a introdução de inovações no mercado quebra a tendência pela estagnação econômica. No entanto, para atingir a inovação, é necessário transpor uma barreira que impede o fluxo livre de conhecimentos científicos para a sua efetiva aplicação.

Na Figura 1 está ilustrada a distinção entre o processo de realimentação de conhecimentos e o processo de inovação. Enquanto o primeiro enriquece a base de conhecimentos, disponibilizando um arcabouço de conhecimentos mais amplo e alargando a fronteira técnocientífica, o segundo, por meio da articulação dos conhecimentos até então acumulados, gera, de forma direta, o desenvolvimento econômico e social por meio da introdução de uma inovação que atende a essas emergências.

Quando se discute sobre os processos de inovação, não se pode deixar de resgatar o modelo linear e o modelo interativo dos mesmos.

O modelo linear (BUSH, 1945), apesar de ter sido base para a formulação de políticas de C\&T durante décadas, apresentava-se deficiente. O seu maior ponto fraco está relacionado ao fato de que os investimentos realizados em pesquisa e desenvolvimento não são necessariamente proporcionais à geração de desenvolvimento. Dessa forma, ganharam espaço novos olhares ao fenômeno da inovação, permitindo, assim, o desenvolvimento de novas teorias que buscavam suprir as deficiências deixadas pelas anteriores.

Até o trabalho intitulado An overview of innovation, de Kline (1986), as abordagens sobre o processo de inovação, que consideravam as inter-relações - tecnológicas e econômicas -, eram muito raras. Como os autores afirmam, enquanto os economistas buscavam analisar a inovação tecnológica como uma "caixa-preta" um sistema contendo componentes e processos desconhecidos, os tecnologistas se preocupavam com o que ocorre dentro da caixa-preta. Esses últimos,

\section{Desenvolvimento econômico}

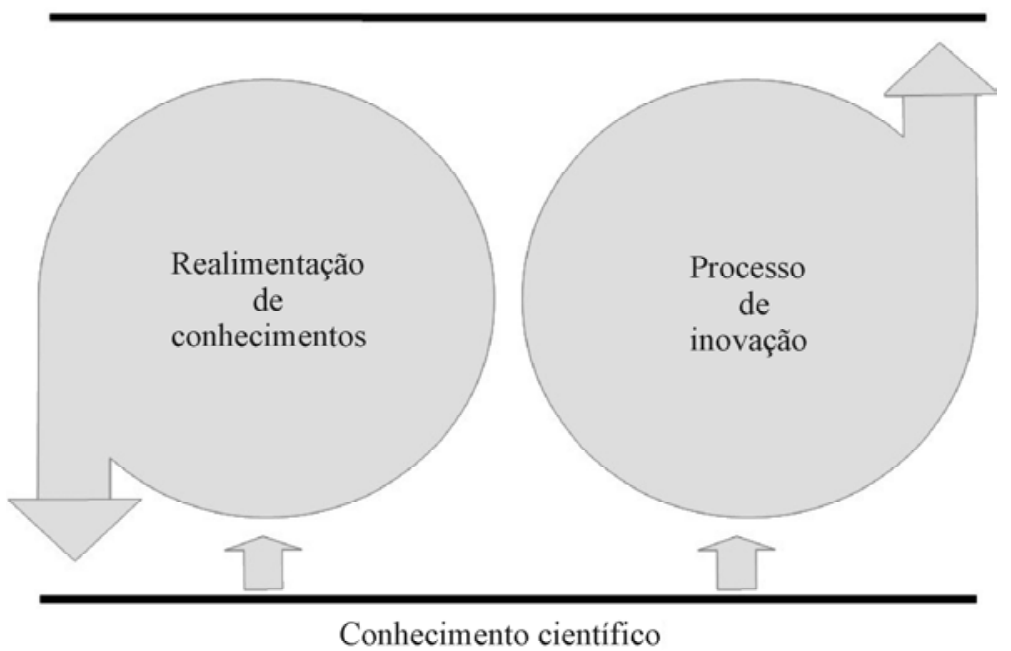

Figura 1 - Distinção entre realimentação de conhecimentos e processo de inovação.

Fonte: desenvolvido pelos autores. 
freqüentemente, negligenciam ou ignoram tanto as forças de mercado, dentro das quais o produto resultante opera, quanto os efeitos resultantes no sistema como um todo pela introdução da inovação.

O propósito de Kline (1986) foi o de preencher essa lacuna ao dedicar um olhar ao processo de inovação, além de focar apenas o que vai dentro da caixa-preta ou focar unicamente os efeitos que as forças externas à organização exercem para o sucesso ou o fracasso de um dado projeto de inovação. Uma crítica de Kline (1986) ao modelo linear está no fato de esse considerar a inovação tecnológica como um ato de produção em lugar de um processo social contínuo, envolvendo atividades de gestão, coordenação, aprendizado, negociação, investigação de necessidades, aquisição de competência, gestão de desenvolvimento de novo produto e gestão financeira, dentre outras.

Para Kline (1986), uma inovação comercial é controlada por duas forças que interagem. De um lado, as forças de mercado, que combinam aspectos demográficos, econômicos e oportunistas e, de outro lado, o avanço da fronteira científica e tecnológica que freqüentemente sugere a possibilidade da introdução de novos produtos ou o desenvolvimento de novos procedimentos capazes de propiciar o aumento de desempenho dos procedimentos existentes ou, mesmo, a possibilidade de produção com menores custos.

Kline (1986) afirmam que o sucesso técnico, ou qualquer forma quantitativa de medir-se o desempenho, após a introdução de uma inovação, é apenas necessário e não uma condição suficiente para estabelecer o seu uso econômico. Em outras palavras, o sucesso técnico de um novo produto, devidamente comprovado e plenamente aceito como tal, não garante a introdução desse novo produto no mercado.

$\mathrm{Na}$ introdução dessas novas formas para se avaliar o sucesso de uma inovação, está a diferença básica entre o modelo linear e o modelo introduzido por Kline (1986). O modelo interativo (Figura 2) combina interações endógenas com interações exógenas - entre os indivíduos e o sistema de ciência e tecnologia mais abrangente. Isso se dá justapondose as necessidades do mercado e o conhecimento científico existente ou por meio da pesquisa científica. Pesquisa e desenvolvimento não são mais a base para a inovação. A abordagem seqüencial é considerada apenas umas das possibilidades para inovar. A relação entre pesquisa científica e tecnologia segue não somente uma direção, mas muitas, interferindo em diversos estágios do processo de inovação.

Pode-se concluir esta parte afirmando que, sob a perspectiva interativa, há a necessidade de se olhar o processo de inovação como um movimento de mudanças, não apenas em parte do sistema, mas nele como um todo. Isso envolve considerar o ambiente de mercado, as facilidades ou dificuldade de produção, o contexto social da organização inovadora, as parcerias entre instituições de pesquisa e empresas, entre outras coisas.

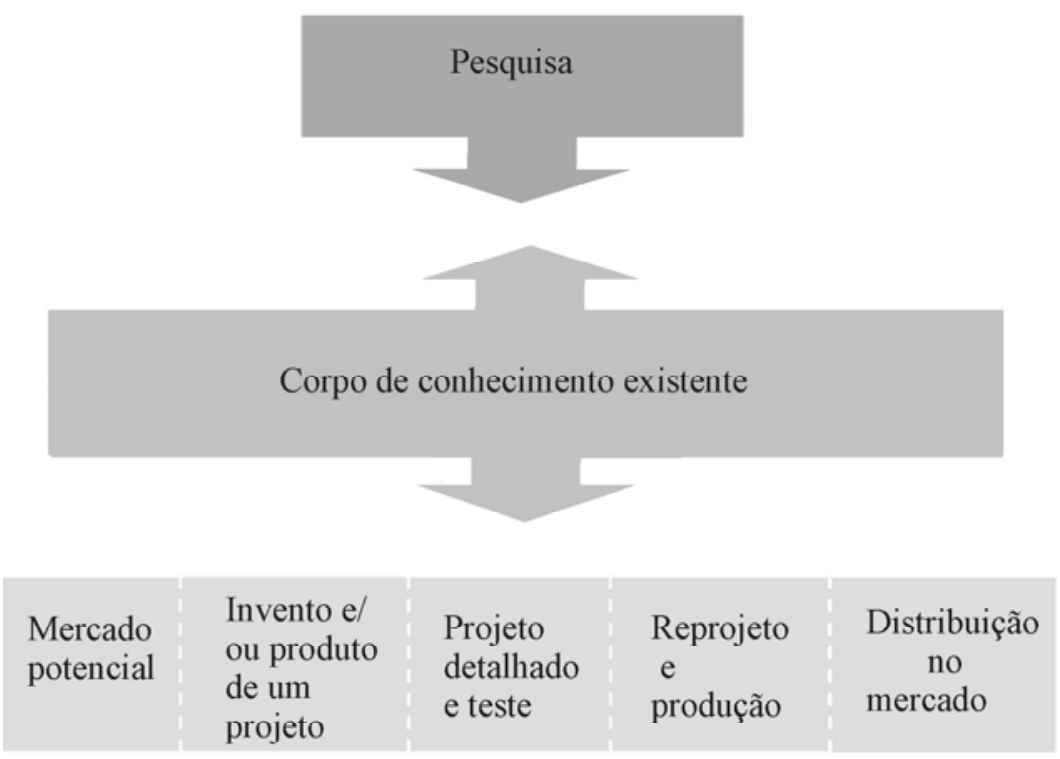

Figura 2 - The chain-linked model.

Fonte: Adaptado de Kline (1986). 


\section{MATERIALE MÉTODOS}

A metodologia adotada por este trabalho foi o estudo de caso, metodologia fundamentada em análises qualitativas. Análises dessa natureza apresentam como principal característica a não utilização de instrumental estatístico para o tratamento dos dados. No entanto, o fato de não apoiar os resultados em técnicas estatísticas não significa que as análises qualitativas sejam especulações subjetivas. É verdade o fato de que a pesquisa qualitativa tem uma dimensão subjetiva maior. Isso, porém, não implica em impossibilidade de se estabelecer procedimentos cientificamente aceitáveis (VIEIRA, 2004).

O estudo de caso consiste de um estudo em profundidade, uma análise intensiva realizada sobre fenômenos reais, reunindo numerosas e detalhadas informações, de forma a apreender a totalidade do fenômeno pesquisado, promovendo a precisão do conhecimento sobre o mesmo. A metodologia do estudo de caso é apropriada para responder a perguntas do tipo "como" e "por que" e pode ser adequada para gerar e construir teoria em uma área sobre a qual há poucos dados (JOIA, 2004).

Este trabalho apresenta o corte seccional com uma perspectiva longitudinal. Sobre este tipo de corte e perspectiva, Vieira (2004, p. 21) definiu:

(...) a coleta de dados será feita em um determinado momento, mas resgata dados e informações de outros períodos passados. O foco está no fenômeno e na forma como se caracteriza no momento da coleta, e os dados resgatados do passado são, normalmente, utilizados para explicitar a configuração atual do fenômeno.

As reflexões teóricas e as proposições apresentadas estimularam a investigação da seguinte questão que serviu como norteadora da pesquisa: que fatores críticos foram essenciais no caso estudado para a aplicação dos conhecimentos desenvolvidos nas pesquisas científicas para o mercado, em forma de inovação tecnológica?

A unidade de análise foi um caso de inovação ocorrido no setor agropecuário de um produto conhecido como Amiréia. O produto é um aperfeiçoamento tecnológico que, por meio da extrusão entre o amido e a uréia, melhoraria a eficiência do aproveitamento dos nutrientes da uréia na alimentação de animais ruminantes. Nesse sentido, pode ser considerada uma inovação incremental (FREEMAN, 1995) ou evolucionária (KLINE, 1986).
O período de realização da pesquisa foi de abril de 2006 a setembro de 2006. No entanto, a entrevista com o principal pesquisador do produto foi realizada em setembro de 2004 e teve dados parciais publicados por Tonelli \& Zambalde (2005). As demais entrevistas foram feitas com pesquisadores, técnicos e investidores da área. No total, foram realizadas cinco entrevistas. Utilizaram-se de roteiros semi-estruturados, construídos de forma a expressar o tipo de participação de cada um no processo estudado, compostos por questões que abordaram desde o surgimento das primeiras pesquisas até o desenvolvimento das técnicas de produção e distribuição do produto no mercado. A pesquisa documental concentrou-se na análise de quatro monografias, uma dissertação de mestrado e vinte e um artigos científicos, todos resultantes de pesquisas sobre o produto. Grande parte dessas pesquisas foi orientada pelo principal entrevistado e, à época, criador de uma empresa destinada a fabricar e distribuir o produto no mercado. Outros materiais, como artigos de jornais e folhetos de divulgação, totalizaram o número de doze documentos.

\section{RESULTADOS E DISCUSSÃO}

Amiréia é um termo que une outros dois: amido e uréia. O produto foi desenvolvido pela ciência zootécnica, na especialidade de nutrição de ruminantes, em torno da hipótese segundo a qual, a junção desses dois ingredientes em um único produto poderia reduzir a toxicidade da uréia pura e aumentar a síntese de proteína, por intermédio do aumento de permanência da uréia no rúmen do animal.

Partindo-se do pressuposto de que a descoberta da utilização da uréia como fonte aproveitável de nutrientes para a alimentação animal tenha sido uma inovação radical (FREEMAN, 1995), a sua utilização inovada por meio da Amiréia é uma inovação incremental.

A evolução das pesquisas, que tornou possível o processo de inovação da Amiréia, está sintetizada na Figura 3. A figura apresenta uma visão da história e trajetória dessa inovação. As pesquisas tiveram início em 1970 e foram desenvolvidas no exterior (EUA). O foco estava em buscar-se um produto que pudesse atender aos objetivos de diminuir a toxicidade da uréia e melhorar a eficiência da absorção de proteínas.

Em meados de 1987, o pesquisador professor Julio César Teixeira trouxe para o Brasil as investigações sobre o tema. O principal objetivo do pesquisador era verificar as potencialidades da Amiréia no país, a partir de experimentos diversos realizados com animais na Universidade Federal de Lavras. 
Desenvolvimento econômico

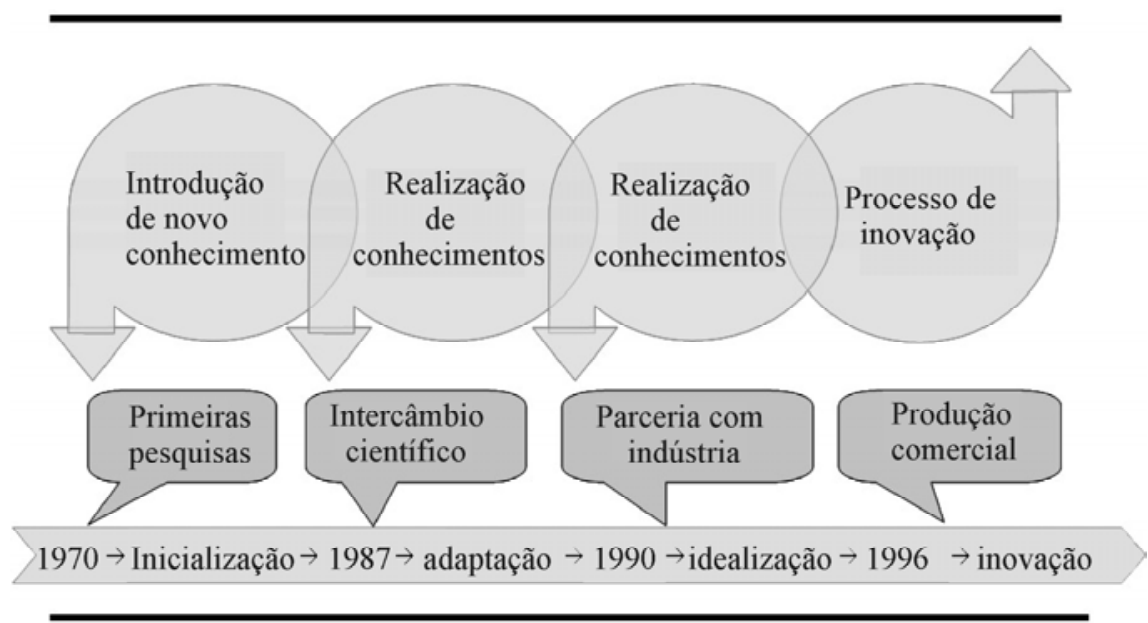

Pesquisa científica

Figura 3 - Evolução longitudinal das pesquisas e o processo de inovação da Amiréia.

Fonte: desenvolvido pelos autores.

A partir de 1990, ocorreu uma parceria com a Petrobrás. A empresa tinha interesse em maximizar as vendas por meio da disseminação do uso de um de seus produtos, ou seja, a uréia. Por meio de contrato, a Petrobrás passou a financiar boa parte das pesquisas. A Universidade, mais especificamente o grupo de pesquisa do qual fazia parte o professor, oferecia recursos humanos e laboratoriais essenciais para alcançar os objetivos da empresa.

As pesquisas geraram por volta de três dezenas de trabalhos, entre os quais os documentos científicos acima citados.

Na Figura 3, visualizam-se as etapas que tornaram possível o processo de inovação da Amiréia. Elas foram as seguintes: a) inicialização: compreendendo o período que vai de 1970 a 1987 , quando as pesquisas foram desenvolvidas exclusivamente no exterior; b) adaptação: período de 1987 a 1990, quando houve a importação dos conhecimentos para o Brasil; c) idealização: compreende o período que vai de 1990 até, aproximadamente, o ano de 2000, quando vigorou uma parceria entre o grupo de pesquisa do principal pesquisador e a Petrobras; d) inovação: a partir de 1996, quando os primeiros investimentos industriais para a produção e venda do novo produto foram realizados, e o produto passou a ser amplamente comercializado em várias partes do país.
Entre o ano de 1970, quando houve a descoberta e o desenvolvimento em laboratório da Amiréia, até o ano de 1996, o conhecimento científico se aprimorou. Nesse período, as diversas pesquisas desenvolvidas a respeito do assunto enriqueceram a base que possibilitou o surgimento do produto. Dessa forma, o processo de inovação esteve estreitamente conectado com o conhecimento prévio vastamente desenvolvido. Apenas por meio desse vasto conhecimento, foi possível encontrar o espaço e o tempo adequados para que a inovação se tornasse uma realidade. Esse foi o primeiro fator crítico do processo de inovação estudado, ou seja, o tempo necessário para a consolidação dos conhecimentos científicos.

O segundo fator crítico identificado como essencial para o sucesso do processo de inovação foi a parceria entre o grupo de pesquisa e a Petrobrás (Universidade/ Empresa) para o financiamento das pesquisas. Na época, com a falta de recursos para pesquisas aplicadas, o apoio de uma empresa do porte de Petrobrás creditava confiança ao projeto. Essa credibilidade foi essencial, não apenas para os futuros investidores e consumidores, que direta ou indiretamente percebiam o endosso de uma grande empresa, como para o pesquisador principal, que percebia no produto uma oportunidade para estabelecer futuros negócios. A despeito dos resultados científicos alcançados, as pesquisas que sucederam essa parceria estiveram muito 
mais próximas do desenvolvimento de uma solução para o mercado.

Os demais elementos analisados levaram à identificação do terceiro fator crítico. Ele está ligado às seguintes constatações: a) A inovação apenas tornou-se viável comercialmente no Brasil; b) não foi encontrada nenhuma pesquisa, cujos resultados fossem relevantes a favor da utilização da Amiréia, publicada em periódicos internacionais ou em revistas científicas nacionais, fora do eixo em torno do qual se desenvolveu grande parte das pesquisas; c) em muitos textos publicados recentemente, afirma-se que a substituição de uréia pela Amiréia não promoveu diferença no consumo e no desempenho dos animais (OLIVEIRA et al., 2004; OLIVEIRAJÚNIOR et al., 2004a,b; VILELA, et al., 2007) e d) o custo da Amiréia é aproximadamente o mesmo, se comparado ao da uréia pura. Dessa forma, torna-se difícil defender a tese de sugerir o aumento da competitividade por meio da introdução da Amiréia, na alimentação dos animais. A maioria dos resultados não confirma esse benefício. Enfim:

“(...) o uso da Amiréia não tem nada a ver com os seus benefícios técnicos, mas, sim, tem a ver com uma questão cultural. Durante muitos anos, os pecuaristas brasileiros - na grande maioria os pequenos, que não recebem a devida orientação profissional - têm desenvolvido o conceito de que a uréia pura leva à contaminação tóxica do rebanho, podendo levar à morte dos animais. (...). Na cabeça dos caras, a uréia pura na ração significa que ela é uma ração é ruim (...). Só no Brasil acontece isso" (Relato de entrevista - pesquisador, 2006).

Como afirmam Kline (1986), o sucesso técnico alcançado, por exemplo, por determinada pesquisa científica, apesar de ser necessário para a introdução de uma inovação no mercado, não é suficiente para estabelecer o seu uso econômico. A própria pesquisa, segundo os autores, pode tornar-se dispensável, uma vez que a inovação, em muitos casos, é o produto da articulação de conhecimentos existentes.

Cabe, portanto, questionar como um processo de inovação foi desencadeado a partir de uma descoberta que não demonstrou claramente nas pesquisas nem desempenho nem benefícios técnicos superiores ao produto até então conhecido no mercado. A explicação está no terceiro fator crítico, ou seja, além da influência dos aspectos econômicos, demográficos e oportunísticos, apontados por Kline (1986), está a influência crítica do aspecto cultural de mercado.

Esse fator exerceu papel fundamental para o sucesso da inovação. A transformação visual do produto quebrou resistências culturais em relação ao consumo da uréia de grande parte dos pecuaristas brasileiros. A respeito disso, deve-se resgatar como se dá o processo de produção. Por meio da extrusão da uréia com o amido, o grânulo de uréia é transformado de uma estrutura cristalina para um pó amarelo. Esse fato passaria despercebido se não fosse um preconceito culturalmente disseminado no Brasil de que a utilização da uréia pura seria considerada perigosa, por parte de muitos pecuaristas, devido ao receio de que ela cause intoxicação nos animais. Na realidade, como afirmam os pesquisadores da área, qualquer utilização indiscriminada - sem que haja o devido controle nutricional - tanto da uréia, quanto da Amiréia, pode, sim, causar intoxicação. O detalhe é que o preconceito só ocorre contra o grânulo cristalino de uréia, facilmente reconhecido na formulação de rações ou compostos. O receio deixou de existir no momento em que não se pôde mais reconhecer a presença da uréia, mesmo que a mesma estivesse presente na sua forma transformada, ou seja, extrusada com o amido.

\section{CONCLUSÕES}

O caso estudado levou à constatação de três fatores críticos para o sucesso no processo de aplicação de conhecimentos científicos produzidos na Universidade. No entanto, não houve a preocupação de indicar a importância relativa de cada fator para esse sucesso. O primeiro deles está relacionado ao fato de que as pesquisas geraram conhecimento prévio. A articulação entre os conhecimentos prévios, necessária em vários estágios do processo de inovação, foi um fator essencial para o sucesso do caso estudado. Esse fato chama a atenção para a importância de se produzir conhecimento básico, além do aplicado, no sentido de criar o fundamento necessário em futuras soluções tecnológicas. Outro fator importante se relaciona com a parceria entre Indústria e o Grupo de Pesquisa. Essa parceria mudou a perspectiva, especialmente do principal pesquisador em relação ao conhecimento que ele e seus colaboradores estavam desenvolvendo, gerando confiança e credibilidade ao projeto, em detrimento dos riscos que a produção comercial de um novo produto gera. A partir de então, as pesquisas deixaram de produzir apenas conhecimento básico e assumiram definitivamente um caráter tecnológico. O terceiro fator crítico está relacionado ao aspecto cultural de mercado, além de outros já apontados por Kline (1986) (aspectos econômicos, demográficos e oportunistas). Esse fator, de forma específica, contribuiu de forma essencial para a adoção pelo mercado da inovação estudada. Isso se verificou em razão da fragilidade da inovação em prover soluções ligadas ao oferecimento de melhor desempenho ou de redução nos custos da atividade pecuária. Apesar de não solucionar definitivamente esses problemas específicos, a inovação quebrou certo tipo de 
resistência cultural dos pecuaristas em relação ao receio de utilizar-se uréia granulada na dieta de seus animais.

\section{REFERÊNCIAS BIBLIOGRÁFICAS}

BRITO, M. J. de. Mudança e cultura organizacional: a construção social de um novo modelo de gestão de P\&D na Embrapa. 2000. 239 f. Tese (Doutorado em administração) Universidade de São Paulo, São Paulo, 2000.

BUSH, V. Science, the endless frontier: a report to the president by Vannevar Bush, director of the office of scientific research and development, july 1945.

Washington, DC: Government Printing Office, 1945.

ETZKOWITZ, H.; LEYDESDORFF, L. Universities in the global economy: a triple helix of university-industrygovernment relations. London: Cassel Academic, 1995.

ETZKOWITZ, H.; LEYDESDORFF, L. The triple helixuniversity-industry-government relations: a laboratory for knowledge-based economic development. European Association Study Science and Technology Review, London, v. 14, n. 1, p. 14-19, 1997.

ETZKOWITZ, H.; LEYDESDORFF, L. The dynamics of innovation: from national systems "mode 2 " to a tripe helix of university-industry-government relations.

Research Policy, Amsterdam, v. 29, n. 2, p. 109-123, 2000.

FREEMAN, C. The national system of innovation in historical perspective. Journal of Economics, London, v. 19, n. 1, p. 5-24, Feb. 1995.

GIBBONS, M. et al. The new production of knowlege: the dynamics of science in contemporary societies. London: Sage, 1994.

JOIA, L. A. Geração de modelos teóricos a partir de estudos de casos múltiplos: da teoria à prática. In:

Pesquisa qualitativa em administração. Rio de Janeiro: FGV, 2004.

KLINE, R. An overview of innovation. In: LANDAU, R.; ROSENBERG, N. The positive sum strategy.

Washington, DC: National Academy, 1986. p. 275-306.

KNOR-CETINA, K. Scientific comunities or transepistemics arenas of research?: a critique of quasi economic models of science. Social Studies of Science, London, v. 12, p. 101-130, 1982.
LATOUR, B.; WOLGAR, S. A vida de laboratório. Rio de Janeiro: Relume-Dumará, 1997.

OLIVEIRA, L. O. F.; SALIBA, E. O. S.; RODRIGUES, N. M.; GONÇALVES, L. C.; BORGES, I.; AMARAL, T. B. Intake and digestibility of Nelore steers grazing pasture and supplemented with multiple mixture. Arquivos Brasileiros de Medicina Veterinária e Zootecnia, Belo Horizonte, v. 56, n. 1, p. 61-68, fev. 2004. Available from: <http:// www.scielo.br/scielo.php?script=sci_arttext $\&$ pid=S010209352004000100010\&lng=en\&nrm=iso >. Access on: 1 ago. 2006.

OLIVEIRA JUNIOR, R. C.; PIRES, A. V.; FERNANDES, J. J. R.; SUSIN, J.; SANTOS, F. A.; ARAÚJO, R. C. Total replacement of soybean meal by urea or Starea in high grain diets on nitrogen metabolism, ruminal ammonia-N concentration and blood parameters in beef cattle.

Revista Brasileira de Zootecnia, Viçosa, v. 33, n. 3, p. 738-748, 2004a. Available from: <http://www.scielo.br/ scielo.php?script=sci_arttext $\&$ pid=S1516$35982004000300023 \& \operatorname{lng}=e n \& n r m=i s o>$. Access on: 1 Aug 2006.

OLIVEIRA JUNIOR, R. C.; PIRES, A. V.; SUSIN, J.; FERNANDES, J. J. R.; SANTOS, F. A.; ARAÚJO, R. C. Digestibilidade de nutrientes em dietas de bovinos contendo uréia ou Amiréia em substituição ao farelo de soja. Pesquisa Agropecuária Brasileira, Brasília, v. 39, n. 2, p. 173-178, May/June 2004b. Disponível em: <http:// www.scielo.br/scielo.php?script=sci_arttext\&pid=S0100204X2004000200011\&lng=pt\&nrm=iso >. Acesso em: 1 ago. 2006.

SHUMPETER, J. A. Capitalismo, socialismo e democracia. [S.l.: s.n.], 1942.

TONELLI, D. F.; ZAMBALDE, A. L. Da pesquisa à inovação: o estudo da trajetória de uma pesquisa até a efetivação de uma inovação. In: ENCONTRO NACIONAL DOS PROGRAMAS DE PÓSGRADUAÇÃO EM ADMINISTRAÇÃO, 29., 2005, Brasília, DF. Anais... Rio de Janeiro: ANPAD, 2005.

VILELA, F. G.; TEIXEIRA, J. C.; PÉREZ, J. R. O.; PAIVA, P. C. DE A.; MUNIZ, J. A.; REIS, S. T. Efeito da substituição do farelo de soja pela amiréia $150 \mathrm{~S}$ no consumo, produção e composição do leite. Ciência e Agrotecnologia, Lavras, V.31, N.5, P.1512-1518, set./out., 2007.

VIEIRA, M. M. F. V. Introdução à pesquisa qualitativa em administração: questões teóricas e epistemológicas. In: _. Pesquisa qualitativa em administração. Rio de Janeiro: FGV, 2004. 